\title{
A SATELLITE-BORNE RADAR WIND SENSOR (RAWS)
}

\author{
Investigators: \\ Richard K. Moore, Michael Stuart, Timothy Propp \\ Radar Systems and Remote Sensing Laboratory \\ University of Kansas Center for Research, Inc. \\ 2291 Irving Hill Road \\ Lawrence, Kansas 66045-2969
}

\section{BACKGROUND}

Modeling global atmospheric circulations and forecasting the weather would improve if worldwide information on winds aloft were available. Accurate prediction of weather is important to agriculture, shipping, air traffic, and many other fields. Global system models of climate are of great importance. Current global atmospheric models use pressure measurements and thermodynamic properties to calculate the effects of wind for use in Numerical Weather Prediction (NWP) models. Inputs to the NWP models are temperature, pressure and wind velocities at different heights. Clearly direct wind measurements could significantly improve the NWP model performance.

The RAdar Wind Sounder (RAWS) program at the University of Kansas is a study of the feasibility and the trade-offs in the design of a space-based radar system to measure wind vectors. This can be done by measuring the Doppler shift of cloud and rain returns from three or more points and calculating the components of the wind vector. The primary tasks related to the RAWS program are to determine:

1. scattering and attenuation models,

2. required radar sensitivity,

3. optimal frequencies,

4. needed antenna size,

5. suitable scan pattern,

6. ways to remove the ambiguity imposed by range and Doppler frequency,

7. spectrum measurements,

8. system configuration,

9. performance as a rain sensor,

10. performance as an ocean-surface wind sensor. 
Xin's dissertation treated all items listed above except items 9-10. He developed a candidate system used throughout the study as a baseline. Our research shows that such an instrument could measure winds at about $1-\mathrm{km}$ height intervals in denser clouds and rain using a large scanning parabolic antenna (8-m diameter in our example), with power comparable to a spaceborne SAR $(6 \mathrm{~kW})$. A conical scan pattern with two pointing angles allows for multiple independent looks at the same observation point. So far the study has treated sensitivity and Doppler measurements in detail.

\section{ACCOMPLISHMENTS FOR THE PAST YEAR}

The RAWS study to date uses the candidate system selected after preliminary study of frequencies and sensitivities. Two frequencies chosen, 10 and $35 \mathrm{GHz}$, allow higher sensitivity for clouds and more penetration for rain. The past year was devoted to modeling the signal-to-noise ratio (SNR) achievable for the two frequencies. The determination of SNR versus cloud penetration depth used a cloud backscattering and attenuation model in the appropriate radar equation. Calculations assumed reasonable losses in reception and transmission, in addition to the atmospheric attenuation. We discovered that ice clouds provide a higher SNR than previously calculated, but some water clouds give lower SNRs than we calculated before.

One of the primary issues in the SNR calculation was the choice of the drop size distribution. Although Xin used several distributions (e.g., log normal, Khrigian and Mazin), this year we used the Deirmendjian cloud model. SNR versus cloud penetration plots were generated to validate the candidate system. Rain, which appears in the cloud models at the lower altitudes, provides ample SNR, as do the higher clouds composed of ice particles. However, in some cloud situations we found the sensitivity for the clouds was marginal or inadequate. At $35 \mathrm{GHz}$, two of the cloud models characterized by 1 to $2 \mathrm{~g} / \mathrm{m}^{3}$ of water content at altitudes extending from 150 to 1500 meters, produced a sufficient SNR. Other models, however, with water contents ranging from 0.5 to $4 \mathrm{~g} / \mathrm{m}^{3}$ and altitudes up to 4000 meters, exhibit SNR of -3 to $-23 \mathrm{~dB}$, largely because of attenuation in the upper cloud layers. These results coupled with the lower SNR at $10 \mathrm{GHz}$, led to an investigation of alternate frequencies. The rain present beneath these clouds provides adequate SNR at $10 \mathrm{GHz}$, and in most cases, at $35 \mathrm{GHz}$. 


\section{CURRENT AND FUTURE RESEARCH}

Analysis is underway to determine the best frequencies for the system. We need a stronger received signal to get a larger-than-unity SNR for all cloud depths. Operating at $94 \mathrm{GHz}$, for example, would increase SNR for clouds with smaller water content at the cost of more attenuation. The use of a $17-$ or $24-\mathrm{GHz}$ frequency would increase the backscatter with a possibly acceptable increase in attenuation.

A larger antenna is under consideration to increase SNR and improve vertical resolution. A rectangular antenna may be used, as this would allow for independent selection of vertical and horizontal beamwidths. The antenna scan pattern is also being reviewed for optimization. With the present conical scan, few individual points will be illuminated for the required three measurements. We will explore an alternate scan or scan rate, such as an adaptive shot pattern, to control slew rates and schedule pulse suppression in regions of low information potential. Such an approach may require use of electronically scanned arrays, which we will also examine.

The Doppler tracker, briefly discussed in Xin's dissertation, will be studied further. This function is critical to achieve the wind speed accuracy required. A phase-lock loop (PLL) will probably be used for this purpose.

The RAWS is potentially a multipurpose instrument: wind aloft, rain, and ocean-surface winds. Once we are more certain of the feasibility of the first use, we plan to examine the other two. Since the wind-aloft task is more demanding than the others, use of RAWS for these applications will be easy. However, such use may require small modifications to the wind-aloft system.

\section{PUBLICATIONS}

Xin, W., "A Satellite Based Radar Wind Sounder," Ph.D. Dissertation, University of Kansas, 1990.

Moore, R. K., M. Stuart, W. Xin \& T. Propp, "A satellite-borne radar Wind sensor (RAWS), presented at IGARSS'92, Houston TX, 25-29 May 1992.

The M.S. thesis by Mr. Timothy Propp is still being written. 


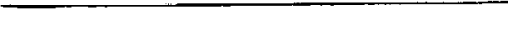

